\title{
A Two-Scale Microfacet Reflectance Model Combining Reflection and Diffraction
}

\author{
NICOLAS HOLZSCHUCH, Inria, Univ. Grenoble-Alpes, CNRS, LJK \\ ROMAIN PACANOWSKI, CNRS, Institut d'Optique Graduate School, University of Bordeaux
}

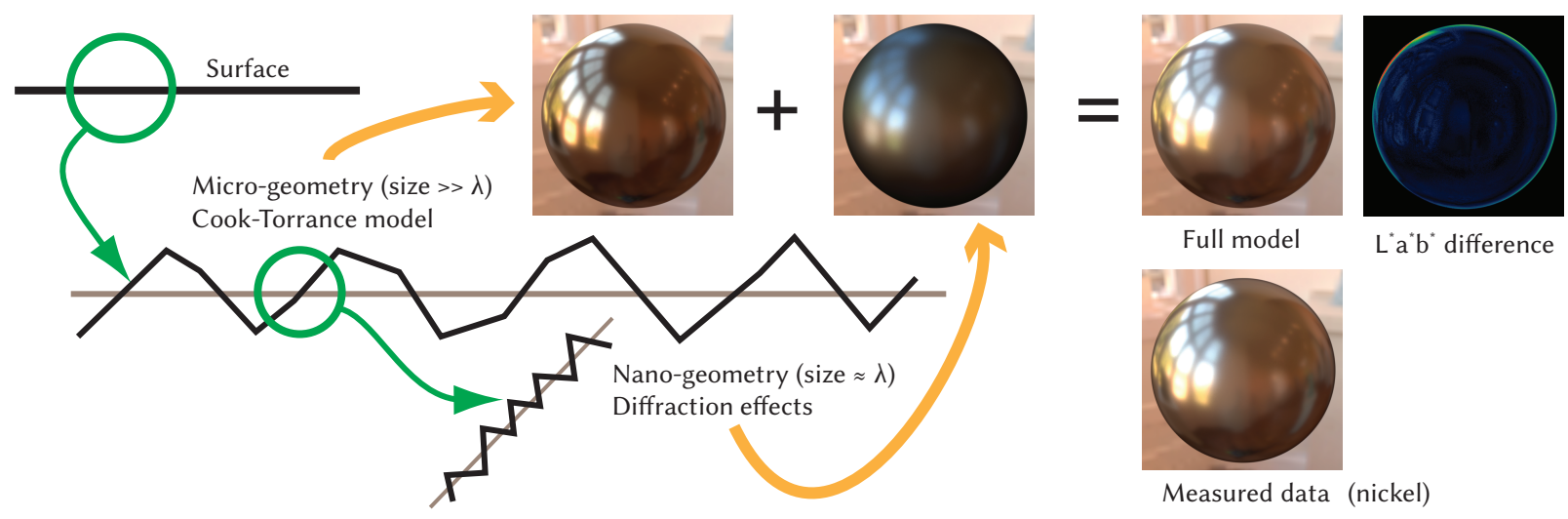

Fig. 1. Material reflectance properties are caused by small variations in surface geometry. We separate these surface variations into micro-geometry, of size larger than the wavelength of visible light, and nano-geometry, of size comparable to the wavelength. The latter produces diffraction effects, with wavelength-dependent effects. The former corresponds to the classical Cook-Torrance lobe. We explain how these two levels interact and show that combined together, they reproduce measured materials faithfully, including subtle color shifts.

\begin{abstract}
Adequate reflectance models are essential for the production of photorealistic images. Microfacet reflectance models predict the appearance of a material at the macroscopic level based on microscopic surface details. They provide a good match with measured reflectance in some cases, but not always. This discrepancy between the behavior predicted by microfacet models and the observed behavior has puzzled researchers for a long time. In this paper, we show that diffraction effects in the micro-geometry provide a plausible explanation. We describe a two-scale reflectance model, separating between geometry details much larger than wavelength and those of size comparable to wavelength. The former model results in the standard Cook-Torrance model. The latter model is responsible for diffraction effects. Diffraction effects at the smaller scale are convolved by the micro-geometry normal distribution. The resulting two-scale model provides a very good approximation to measured reflectances.
\end{abstract}

CCS Concepts: • Computing methodologies $\rightarrow$ Reflectance modeling;

Additional Key Words and Phrases: material models, BRDF, diffraction

ACM Reference format:

Nicolas Holzschuch and Romain Pacanowski. 2017. A Two-Scale Microfacet Reflectance Model Combining Reflection and Diffraction. ACM Trans. Graph. 36, 4, Article 66 (July 2017), 12 pages.

DOI: http://dx.doi.org/10.1145/3072959.3073621

This work was supported in part by an ANR grant ANR-15-CE38-0005 "MATERIAL". Permission to make digital or hard copies of all or part of this work for personal or classroom use is granted without fee provided that copies are not made or distributed for profit or commercial advantage and that copies bear this notice and the full citation on the first page. Copyrights for components of this work owned by others than ACM must be honored. Abstracting with credit is permitted. To copy otherwise, or republish, to post on servers or to redistribute to lists, requires prior specific permission and/or a fee. Request permissions from permissions@acm.org.

(c) 2017 ACM. 0730-0301/2017/7-ART66 \$15.00

DOI: http://dx.doi.org/10.1145/3072959.3073621

\section{INTRODUCTION}

Reflectance properties express how a material interacts with light. They are responsible for the visual appearance of objects in virtual scenes, e.g., whether they look shiny, metallic, or plastic. Microfacet reflectance models are widely used in computer graphics, both in research and industry. Based on a surface geometric model at the microscopic level, they predict the overall material aspect at the macroscopic level. The connection between surface geometry and material aspect makes it simpler for users to edit materials, e.g., making them shinier by decreasing the roughness.

The most commonly used microfacet model is the Cook-Torrance model [1982]. It assumes that microfacets are made of a specular material, and that they are much larger than the wavelength of light, so that the surface response is defined by optical geometry. The main contributing parameter is the normal distribution function (NDF) of the microfacets. At the other end of the spectrum, the diffraction model assumes that surface micro-geometry is comparable in size with light wavelengths, and predicts material response caused by diffraction effects due to variations in the optical path length.

Both models provide relatively good fits with measured reflectance properties for some aspects. However, measured reflectances can exhibit behaviors that are in contradiction with predictions of either model. First, the Cook-Torrance model provides a good prediction of the lobe shape near the specular peak [Ngan et al. 2005] but for some measured materials, the lobe width varies at different wavelengths, contradicting the idea that a single geometry can capture the entire material response [Butler et al. 2015b]. The shape of the lobe away from the specular peak corresponds to the prediction of 
the diffraction model [Löw et al. 2012] but you obtain a better fit by removing the wavelength dependency from the model. Summing two microfacet lobes often results in a better fit [Cook and Torrance 1982; Ngan et al. 2005; Burley 2012; Löw et al. 2012], but this is also in contradiction with the physical model, as there cannot be two normal distributions for a single microgeometry.

In this paper, we present a new surface reflectance model, based on the following hypothesis: surface geometry details are present at all scales, from the size of the wavelength of incoming light to much larger. We separate the surface geometry into two scale levels: details much larger than wavelength, which we call microgeometry, and details of the same order of magnitude as wavelength, which we call nano-geometry. Each micro-geometry carries smaller nano-geometry details (see Figure 1). The material response is a combination of effects at the micro- and nano- geometry levels, taking into account reflection and diffraction. We express the overall material response as a sum of a standard Cook-Torrance lobe and a Cook-Torrance-Diffraction lobe. The latter is a convolution of the Cook-Torrance and diffraction responses. This new model provides a good fit with measured reflectance, for a wide range of materials, and explains several effects that were previously impossible to explain, such as wavelength dependency and behaviour at grazing angles.

All parameters in our model are connected to physical quantities: the color properties of the material come from its index of refraction. The micro-geometry is defined by its normal distribution function; we use a Exponential Power Distribution NDF, a new model with two parameters. The nano-geometry is defined by its Power Spectrum and the variance of its surface height.

In the next section, we review previous work on material reflectance, as well as issues raised when comparing measured reflectance with model predictions. In Section 3 we provide a quick description of the Cook-Torrance reflection model and the Modified Harvey-Shack model for diffraction. In Section 4, we describe our two-scale model and its properties. In Section 5, we show that our model provides a good approximation to a wide range of measured materials; we also compare our model with other reflectance models. Our model provides a better approximation of measured material properties while being physically consistent. Finally, we conclude and present avenues for future work in Section 6.

\section{PREVIOUS WORK}

\subsection{Microfacet Model}

The microfacet model [Torrance and Sparrow 1967; Cook and Torrance 1982] assumes that surface micro-geometry is made of microfacets. It predicts the overall surface appearance from the probability distribution of these microfacet normals. Standard distribution models include Gaussian [Cook and Torrance 1982], rational fraction [Trowbridge and Reitz 1975; Walter et al. 2007], fraction to the power $p$ [Löw et al. 2012; Burley 2012], Shifted-Gamma [Bagher et al. 2012] and exponential of a power function [Brady et al. 2014]. Heitz [2014b] explains that a good normal distribution should be shape-invariant to be compatible with linear scaling operations.

The shadowing and masking term is essential for energy conservation in the microfacet model. Assuming that positions and orientations of microfacets are independent, Smith [1967] computes a shadowing/masking term from two successive integrations of the slope distribution. Heitz [2014b] shows that this is the most physically consistent method to compute the shadowing and masking term, and provides an improved shadowing term taking into account correlation between input and output directions.

Heitz [2014a] designed a multi-scale microfacet BRDF model, combining two Cook-Torrance lobes at different scales. The difference with our approach is that we combine lobes from two different physical phenomena.

\subsection{Diffraction Models}

Diffraction models focus on diffraction effects caused by variations in surface height. They are widely used in the optical engineering community, for example, to measure imperfections on smooth surfaces like telescope mirrors. Several models express the relationship between surface height distribution and reflectance properties: The Rayleigh-Rice vector perturbation theory is designed for smooth surfaces and wide-angle scattering. The Beckmann-Kirchhoff [Beckmann and Spizzichino 1987] and Modified Beckmann-Kirchhoff [Vernold and Harvey 1998] theories represent the BRDF as an infinite sum; they are valid for a wide range of surfaces, but for small light and view incident angles. For very smooth or very rough surfaces, the infinite sum is approximated by a single term. The Harvey-Shack model [Harvey 1975] expresses diffraction effects using a surface transfer function. For smooth surfaces, this transfer function is the Power Spectral Distribution of surface height. Krywonos [2006] extends this with the Generalized Harvey-Shack model, where the surface transfer function is recomputed for each incoming direction. This model is valid for all surfaces, rough and smooth, and for all angles; it provides a better approximation of measured reflectances than other diffraction models.

He et al. [1991] use the Beckmann-Kirchhoff theory to build a diffraction-based reflectance model for isotropic surfaces. It is not widely used for rendering and fitting, as it is computationally expensive. This model is extended to anisotropy by Stam [1999].

Löw et al. [2012] introduce the Harvey-Shack diffraction model to the computer graphics community but they left out several elements such as wavelength dependency, combination with specular reflection, and color term, We reintroduce them in this paper.

In this paper, we build on the Modified Harvey-Shack model, as it models diffraction effects using a single term instead of an infinite sum and has been shown to agree better with actual reflectance measurements.

\subsection{Comparison with Measured Reflectances}

Butler et al. studied the connection between the microfacet model and diffractions models. They found that measured reflectance functions are well approximated by the microfacet model in the incident plane [Butler et al. 2015a], but that the normal distribution appears to change with the wavelength [Butler et al. 2015b].

The two models have different parameters and predict different shapes for reflectance lobes outside the incident plane. Löw et al. [2012] showed that the shape of the reflectance lobe in measured materials corresponds to what diffraction theory predicts and that their simplified diffraction model provides a good fit with measured reflectances. 
Matusik et al. [2003] released reflectance measurements for a large range of materials; we use their database in our tests. Ngan et al. [2005] fit parametric BRDF models to these measured data. They found the best fits for the Cook-Torrance [1982] and Lafortune et al. [1997] models, except at grazing angles where measured properties differ strongly with model predictions. They also reported that many specular materials are poorly approximated with a single lobe. The quality of the fits improves with multiple lobes, but the fitting process becomes unstable.

Ashikhmin and Premože [2007] approximated measured BRDFs using retro-reflection: if input and output directions are equal, the entire BRDF can be expressed as a function of the half-vector. By storing this function they get a compact BRDF model, that fits measured data very well.

Bagher et al. [2016] compute the normal distribution and Fresnel term directly by fitting on the entire data. They use separate parameters for each color channel, in contradiction with the physical model. They report a better fit by treating the shadowing function as a separate parameter instead of computing it with Smith [1967] method. In this paper, we provide an explanation for this observed discrepancy between physical models and measured data at grazing angles.

Brady et al. [2014] applied genetic programming to discover new analytical BRDF models from measured BRDFs. We use a distribution that is similar to the one they found, but with shape-invariance property. Our contributions include the normalization constant, the Smith shadowing term and importance sampling method for this distribution.

Holzschuch and Pacanowski [2015b], through an empirical study, show that measured reflectances appear to be a combination of the two phenomena: diffraction for wide-angle scattering, microfacet reflection close to the specular direction. In later work [2015a; 2016], they show that summing the two components provides a good approximation to all measured reflectances. Compared to their work, we provide a physical explanation for the relationship between the two lobes, as well as explicit convolution computation. We also provide a better fit with measured materials while reducing the number of parameters.

\section{BACKGROUND}

\subsection{Microfacet Model}

The microfacet model [Cook and Torrance 1982] is widely used in computer graphics. It is based on the hypothesis that surface details are much larger than the wavelengths of light, so the rules of geometrical optics apply. The model has been described in great details in the literature [Cook and Torrance 1982; Walter et al. 2007; Heitz 2014b]. We focus here on key points.

The model assumes that the surface is made of microfacets oriented according a normal distribution function $D$. Each microfacet acts as a specular mirror; its reflectance is a Dirac delta function, multiplied by the Fresnel term for reflection:

$$
\rho_{\mu F}(\boldsymbol{i}, \boldsymbol{o})=F(\boldsymbol{i}, \boldsymbol{o}) \frac{\delta(\operatorname{refl}(\boldsymbol{i}), \boldsymbol{o})}{\cos \theta_{\boldsymbol{o}}} .
$$

The Fresnel term $F$ is responsible for the color of the microfacet. It depends on the index of refraction $\eta(\lambda)$ of the material; the index of

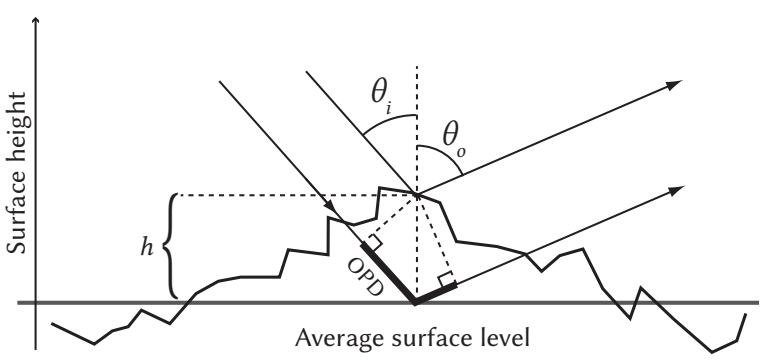

Fig. 2. Light reflected off a surface at a height $h$ has travelled a shorter distance. This Optical Path Difference (OPD) causes interference between parallel rays and is responsible for diffraction effects. Here, $O P D=$ $\left(\cos \theta_{i}+\cos \theta_{o}\right) h(x, y)$ [Krywonos 2006]. Heights $h$ are relative to the average surface level.

refraction depends on the wavelength $\lambda$. For dielectrics (transparent materials), $\eta$ is a real number, related to the speed of light inside the material. For conductors, $\eta$ is a complex number, with real and imaginary parts: $\eta(\lambda)=n(\lambda)+\mathrm{i} k(\lambda)$.

Summing the contributions of all microfacets results in the CookTorrance reflectance model:

$$
\rho_{C T}(\boldsymbol{i}, \boldsymbol{o})=\frac{F\left(\eta, \theta_{d}\right) D\left(\theta_{h}\right) G(\boldsymbol{i}, \boldsymbol{o})}{4 \cos \theta_{i} \cos \theta_{\boldsymbol{o}}} .
$$

The shadowing-masking term $G$ expresses the probability for light to be blocked before or after the specular reflection on the microfacet. It is usually computed using Smith's method through a double integration of $D$ [Smith 1967; Walter et al. 2007; Heitz 2014b].

Note that $D$ and $G$ only depend on the geometry of microfacets, not on the wavelength of the incoming light. Reflection lobes for the different wavelengths are scaled version of each other, with the same relative width. The only wavelength dependency comes from the Fresnel term $F$, which is responsible for the material color. For unpolarized light, $F$ is defined by:

$$
\begin{aligned}
c_{i} & =\left(\eta^{2}-\sin ^{2} \theta\right)^{\frac{1}{2}} & F(\theta) & =\frac{F_{s}(\theta)+F_{p}(\theta)}{2} \\
F_{S}(\theta) & =\left|\frac{\cos \theta-c_{i}}{\cos \theta+c_{i}}\right|^{2} & F_{p}(\theta) & =\left|\frac{\eta^{2} \cos \theta-c_{i}}{\eta^{2} \cos \theta+c_{i}}\right|^{2} .
\end{aligned}
$$

\subsection{Diffraction: Modified Harvey-Shack Theory}

We review the basis of diffraction theory for predicting material reflectance, focusing on the Harvey-Shack theory, as it is applicable to a wide range of configurations and provides a good approximation to measured reflectance. The theory is based on the distance travelled by light as it is reflected on the surface. Because the surface is not flat, light reflected at a point of height $h(x, y)$ has travelled a shorter distance than if it was reflected at the reference height (see Figure 2) [Krywonos 2006, p. 163]. This difference in optical path length (OPD):

$$
O P D=\left(\cos \theta_{i}+\cos \theta_{o}\right) h(x, y)
$$

results in phase variations at the reflected wavefront of $(2 \pi / \lambda)\left(\cos \theta_{i}+\right.$ $\left.\cos \theta_{o}\right) h(x, y)$. Averaging these phase variations gives the reflectance function. 


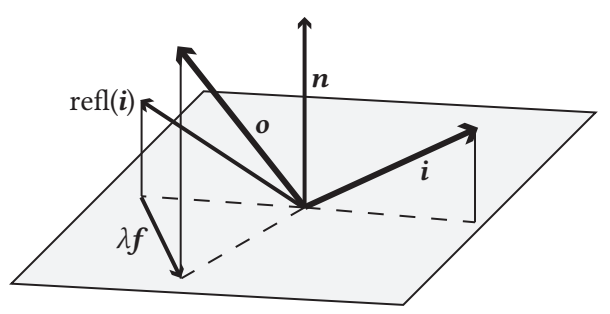

Fig. 3. Diffraction effects depend on $f$, projected vector between reflected incoming direction $\operatorname{refl}(\boldsymbol{i})$ and outgoing direction $\boldsymbol{o}$, divided by the wavelength $\lambda$.

Reflectance $\rho_{\text {diffr. }}$ caused by surface variations is separated in two different lobes: a Dirac in the specular reflection direction, surrounded by a halo of scattered light [Harvey 1975; Krywonos 2006]. Their relative intensity depends on the surface roughness $\sigma_{s}^{2}$, defined as the variance of height distribution $h$ :

$$
\begin{aligned}
\rho_{\text {diffr. }}(\boldsymbol{i}, \boldsymbol{o}) & =A F(\boldsymbol{i}, \boldsymbol{o}) \frac{\delta(\operatorname{refl}(\boldsymbol{i}), \boldsymbol{o})}{\cos \theta_{\boldsymbol{o}}}+(1-A) Q(\boldsymbol{i}, \boldsymbol{o}) S_{H S}(\boldsymbol{f}) \\
\text { with } A & =\mathrm{e}^{-\left(2 \pi \frac{\sigma_{S}}{\lambda}\left(\cos \theta_{i}+\cos \theta_{\boldsymbol{o}}\right)\right)^{2}},
\end{aligned}
$$

where $S_{H S}$ is a scattering function, $F$ is the Fresnel term for reflectance, defined in Equation 4 and $Q$ is the color term for diffraction, defined in Section 3.2.1.

As the diffraction lobe increases, the specular peak decreases. Their relative importance depends on surface roughness and directions: at grazing angles, $\cos \theta_{i} \approx 0$ and $A$ is almost equal to 1 ; most of the energy goes to the specular reflection. The influence of diffraction is stronger at normal incidence, where $\cos \theta_{i} \approx 1$.

The main parameter controlling the diffraction lobe is the $2 \mathrm{D}$ vector $f$, built from the projections on the tangent plane of the difference between the specular reflection of incoming direction and the outgoing direction, divided by the wavelength (see Figure 3):

$$
\begin{aligned}
& f=\frac{1}{\lambda} \operatorname{proj}(\operatorname{refl}(\boldsymbol{i})-\boldsymbol{o})=\frac{1}{\lambda}((\boldsymbol{i}+\boldsymbol{o}) \times \boldsymbol{n}) \times \boldsymbol{n} \\
& f=\|f\|=\frac{2}{\lambda} \sin \theta_{h} \cos \theta_{d} .
\end{aligned}
$$

3.2.1 Color and Polarization. The color of the diffraction lobe comes from $Q(\boldsymbol{i}, \boldsymbol{o})$, a term dependent on the index of refraction, different from the Fresnel term for reflection, $F$, defined in Equation 4. $Q$ comes from the Rayleigh-Rice theory of diffraction for smooth surfaces [Krywonos 2006, p. 34]. Harvey-Shack diffraction is a scalar theory, unable to account for polarization effects. Krywonos suggests multiplying its prediction by the same $Q$ factor [Krywonos 2006, p. 178] to account for polarization effects.

$Q$ depends on the azimuthal angle $\phi$ between reflected incoming direction and outgoing direction. We write $\left(\theta_{i}, \phi_{i}\right)$ and $\left(\theta_{o}, \phi_{o}\right)$ the spherical coordinates of unit vectors $\boldsymbol{i}$ and $\boldsymbol{o} . Q$ is defined as:

$$
\begin{aligned}
& \phi=\phi_{o}-\phi_{i}-\pi, \quad c_{i}=\left(\eta^{2}-\sin ^{2} \theta_{i}\right)^{\frac{1}{2}}, \quad c_{o}=\left(\eta^{2}-\sin ^{2} \theta_{o}\right)^{\frac{1}{2}} \\
& q_{0}=\left(\eta^{2}-1\right)\left(\cos \theta_{i}+c_{i}\right)^{-1} \quad q_{1}=\left(\eta^{2}-1\right)\left(\eta^{2} \cos \theta_{i}+c_{i}\right)^{-1} \\
& q_{2}=\left(\cos \theta_{o}+c_{o}\right)^{-1} \quad q_{3}=\left(\eta^{2} \cos \theta_{o}+c_{o}\right)^{-1} \\
& Q_{s s}=q_{0} q_{2} \cos \phi \quad Q_{s p}=q_{0} q_{3} c_{o} \sin \phi \quad Q_{p s}=q_{1} q_{2} c_{i} \sin \phi \\
& Q_{p p}=q_{1} q_{3}\left(c_{i} c_{o} \cos \phi-\eta^{2} \sin \theta_{i} \sin \theta_{o}\right) \\
& Q=\left|Q_{s s}\right|^{2}+\left|Q_{s p}\right|^{2}+\left|Q_{p s}\right|^{2}+\left|Q_{p p}\right|^{2},
\end{aligned}
$$

$Q$ includes cross-polarization transfer: part of the parallel-polarized light is reflected as perpendicular-polarized, and reciprocally, as expressed by $Q_{s p}$ and $Q_{p s}$.

Along the specular reflected direction, where $\theta_{i}=\theta_{o}$ and $\phi_{o}=$ $\phi_{i}+\pi$, we have $Q_{s p}=0, Q_{p s}=0$, and $Q=2 F$. Outside of this specific case, the two color terms are different.

3.2.2 Smooth Surface Linearization. In this paper, we use the exponential expression for $A$ (Equation 7). Other researchers use the smooth-surface approximation, where:

$$
\rho_{\text {diffr. }}(\boldsymbol{i}, \boldsymbol{o})=\frac{4 \pi^{2}}{\lambda^{4}}\left(\cos \theta_{i}+\cos \theta_{o}\right)^{2} \operatorname{PSD}(f) .
$$

The two expressions are connected. Equation 11 is derived from Equations 6 and 7, assuming surface details are much smaller than light wavelength: $\sigma_{s} / \lambda \ll 1$. Using a linear approximation:

$$
A \approx 1-\left(2 \pi \frac{\sigma_{s}}{\lambda}\left(\cos \theta_{i}+\cos \theta_{o}\right)\right)^{2},
$$

the diffraction lobe becomes:

$$
\rho_{\text {diffr. }}(\boldsymbol{i}, \boldsymbol{o})=4 \pi^{2} \frac{\sigma_{s}^{2}}{\lambda^{2}}\left(\cos \theta_{i}+\cos \theta_{o}\right)^{2} S_{H S}(f) .
$$

The scattering function $S_{H S}$ is expressed with a scaled version of the Power Spectral Density (PSD) of the surface, giving Equation 11, the formula for Generalized Harvey-Shack Diffraction for smooth surfaces [Krywonos 2006, p. 167]. The earlier Modified Harvey-Shack theory uses a similar derivation with a different expression for the Optical Path Difference: $O P D=2 \cos \theta_{i} h(x, y)$. Both models depend on the wavelength in two places: the intensity of the diffraction lobe decreases with $1 / \lambda^{4}$ and the width of the lobe increases with $\lambda$, since $f$ contains a $1 / \lambda$ term.

The linearization in Equation 12 is only valid for very smooth surfaces $\left(\sigma_{s} \ll \lambda\right)$. We use the exponential expression (Equation 7) as it is valid over a larger range [Harvey et al. 2012], even though we focus on small surface variations $\left(\sigma_{s}<\lambda\right)$.

3.2.3 K-correlation Model. If the spatial autocovariance function of the surface height is a Gaussian, $S_{H S}$ is also a Gaussian, simplifying computations. Most optical surfaces, however, do not have a Gaussian autocovariance function. They tend to have an inverse power law falloff at large spatial frequencies. For these surfaces, the K-correlation model, or abc model [Hoenders et al. 1979; Church and Takacs 1986], is widely used:

$$
S_{H S}(f)=\frac{1}{2 \sqrt{\pi}} \frac{\Gamma((c+1) / 2)}{\Gamma(c / 2)} \frac{a b}{\left(1+b^{2} f^{2}\right)^{\frac{c+1}{2}}},
$$




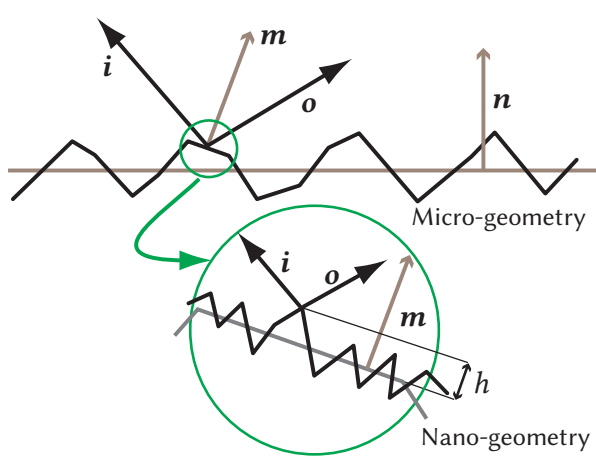

Fig. 4. Surface details happen at all scales. We organize them into microgeometry, with size much larger than visible light wavelengths, and nanogeometry, with size comparable to wavelength. Nano-geometry height $h$ is measured relative to the underlying micro-geometry.

where $\Gamma$ is the Gamma function. Integrating this distribution gives the surface roughness $\sigma_{s}^{2}$. It is finite only for $c>1$ and is equal to:

$$
\sigma_{s}^{2}=\frac{\sqrt{\pi}}{c-1} \frac{\Gamma((c+1) / 2)}{\Gamma(c / 2)} \frac{a}{b} .
$$

We use this expression to get a simpler formula for $S_{H S}$ :

$$
S_{H S}(f)=\frac{c-1}{2 \pi} \frac{\sigma_{s}^{2} b^{2}}{\left(1+b^{2} f^{2}\right)^{\frac{c+1}{2}}} .
$$

3.2.4 Renormalization. The scattering function $S_{H S}$ used in Equation 7 is defined over the entire 2D plane for its parameter, $f$. But $f$ is limited in its variation, as the projections of incoming and outgoing directions must fall over the unit disc in the plane. For energy conservation, $S_{H S}$ must be renormalized by dividing by the integral over the set of possible values for $f$ :

$$
\begin{aligned}
\hat{S}_{H S} & =\frac{\sigma_{s}^{2}}{\sigma_{\text {rel }}^{2}} S_{H S} \\
\sigma_{s}^{2} & =\int_{-\infty}^{\infty} \int_{-\infty}^{\infty} S_{H S}(f) \mathrm{d} f_{x} \mathrm{~d} f_{y} \\
\sigma_{\text {rel }}^{2}\left(\theta_{i}\right) & =\int_{\|\boldsymbol{o}\| \leq 1} S_{H S}(f) \mathrm{d} f_{x} \mathrm{~d} f_{y} .
\end{aligned}
$$

This renormalization ensures that the energy of the diffraction lobe is constant. The same renormalization constant $\sigma_{\text {rel }}$ is also used for energy repartition between specular and diffraction lobes [Harvey et al. 2012]:

$$
A=\mathrm{e}^{-\left(2 \pi \frac{\sigma_{\mathrm{rel}}}{\lambda}\left(\cos \theta_{i}+\cos \theta_{o}\right)\right)^{2}} .
$$

In practice, we precompute the renormalization ratio $\sigma_{\text {rel }} / \sigma_{s}$ for the K-correlation model, for a large range of values for $\theta_{i}, b$ and $c$, and store it in a $8.9 \mathrm{MB}$ table (provided in companion material).

\section{OUR TWO-SCALE BRDF MODEL}

Our model is based on the following observation: we cannot assume that all surface micro-geometry will be much larger than light wavelengths, as with the Cook-Torrance model, or of the same order of magnitude, as with the Harvey-Shack model. Surface micro-details are likely to happen at all scales, from nanometers to micrometers.

We separate surface details in two groups: large-scale details, which we call micro-geometry, corresponding to surface details much larger than the light wavelengths, and small-scale details, which we call nano-geometry, of size similar to light wavelengths. Small-scale details are defined relatively to large-scale details: height variations caused by nano-geometry are measured based on the underlying microfacet (see Figure 4).

The reflectance of each microfacet is given by the diffraction model, described in Section 3.2, depending on the nano-geometry. Overall material reflectance is given by combining each microfacet reflectance together.

\subsection{Generic Two-Scale Model}

The original microfacet framework is generic enough to work with any kind of reflectance on the microfacets. We take the formulation from Walter et al. [2007]: the macro-surface has normal $\boldsymbol{n}$; each individual microfacet, with reflectance $f_{s}$, has normal $\boldsymbol{m}$ following a normal distribution $D(\boldsymbol{m})$. The overall reflectance for the surface is the integral of the contributions from each microfacet, averaged by the probability for each microfacet orientation:

$$
\rho(\boldsymbol{i}, \boldsymbol{o})=\int_{\Omega}\left|\frac{\boldsymbol{i} \cdot \boldsymbol{m}}{\boldsymbol{i} \cdot \boldsymbol{n}}\right| f_{s}(\boldsymbol{i}, \boldsymbol{o}, \boldsymbol{m})\left|\frac{\boldsymbol{o} \cdot \boldsymbol{m}}{\boldsymbol{o} \cdot \boldsymbol{n}}\right| G(\boldsymbol{i}, \boldsymbol{o}) D(\boldsymbol{m}) \mathrm{d} \omega_{m} .
$$

In the generic case, there is no closed-form solution to this equation. If $f_{s}$ is a Dirac, the integral collapses into a function evaluation and we get the standard Cook-Torrance form.

We assume that $f_{s}$ comes from diffraction effects, due to nanoscale imperfections on each microfacet:

$$
\begin{aligned}
& f_{S}(\boldsymbol{i}, \boldsymbol{o}, \boldsymbol{m})=A F(\boldsymbol{i}, \boldsymbol{o}) \delta(\operatorname{refl}(\boldsymbol{i}), \boldsymbol{o})+(1-A) Q(\boldsymbol{i}, \boldsymbol{o}) S_{H S}(\boldsymbol{f}) \\
& \text { with } A=\mathrm{e}^{-\left(2 \pi \frac{\sigma_{\mathrm{rel}}}{\lambda}(\boldsymbol{i} \cdot \boldsymbol{m}+\boldsymbol{o} \cdot \boldsymbol{m})\right)^{2}} \text {. }
\end{aligned}
$$

The first term contains a Dirac, so we get the standard Cook-Torrance lobe, multiplied by $A$ evaluated where $\operatorname{refl}(\boldsymbol{i})=\boldsymbol{o}$, or $\boldsymbol{m}=\boldsymbol{h}$. The combined two-scale reflectance is the sum of a Cook-Torrance lobe multiplied by $A_{\text {spec }}\left(\theta_{d}\right)$, and of a combined Cook-Torrance diffraction lobe, $\rho_{C T D}$ :

$$
\begin{aligned}
& \rho(\boldsymbol{i}, \boldsymbol{o})=A_{\mathrm{spec}}\left(\theta_{d}\right) \rho_{C T}(\boldsymbol{i}, \boldsymbol{o})+\rho_{C T D}(\boldsymbol{i}, \boldsymbol{o}) \\
& \text { where } A_{\mathrm{spec}}\left(\theta_{d}\right)=\mathrm{e}^{-\left(2 \pi \frac{\sigma_{\mathrm{rel}}}{\lambda}\left(2 \cos \theta_{d}\right)\right)^{2}} \\
& \rho_{C T}(\boldsymbol{i}, \boldsymbol{o})=\frac{F\left(\eta, \theta_{d}\right) D\left(\theta_{h}\right) G(\boldsymbol{i}, \boldsymbol{o})}{4 \cos \theta_{i} \cos \theta_{o}} \\
& \rho_{C T D}(\boldsymbol{i}, \boldsymbol{o})= \\
& \int_{\Omega}\left|\frac{\boldsymbol{i} \cdot \boldsymbol{m}}{\boldsymbol{i} \cdot \boldsymbol{n}}\right|(1-A) Q(\boldsymbol{i}, \boldsymbol{o}) S_{H S}(\boldsymbol{f})\left|\frac{\boldsymbol{o} \cdot \boldsymbol{m}}{\boldsymbol{o} \cdot \boldsymbol{n}}\right| G(\boldsymbol{i}, \boldsymbol{o}) D(\boldsymbol{m}) \mathrm{d} \omega_{m} .
\end{aligned}
$$

\subsection{Evaluating the Cook-Torrance Diffraction Lobe}

There is no closed-form version for the Cook-Torrance diffraction lobe $\rho_{C T D}$. Here, we show it can be approximated by a convolution by neglecting the variations of some terms in the integral. In Section 4.4, we give an approximate form for this convolution under certain assumptions on the $D$ and $S_{H S}$ functions. 


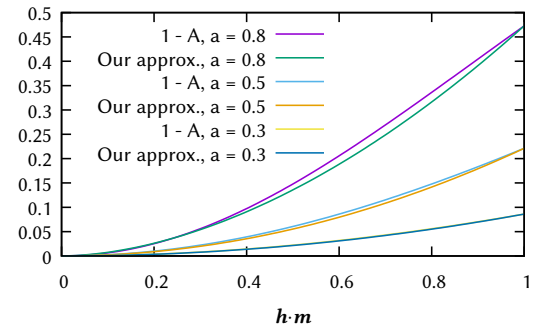

(a) Quality of $1-A \approx\left(1-A_{\text {spec }}\left(\theta_{d}\right)\right)(\boldsymbol{h} \cdot \boldsymbol{m})^{2}$, depending on $a=2 \pi\left(2 \cos \theta_{d}\right) \sigma_{\text {rel }} / \lambda$.
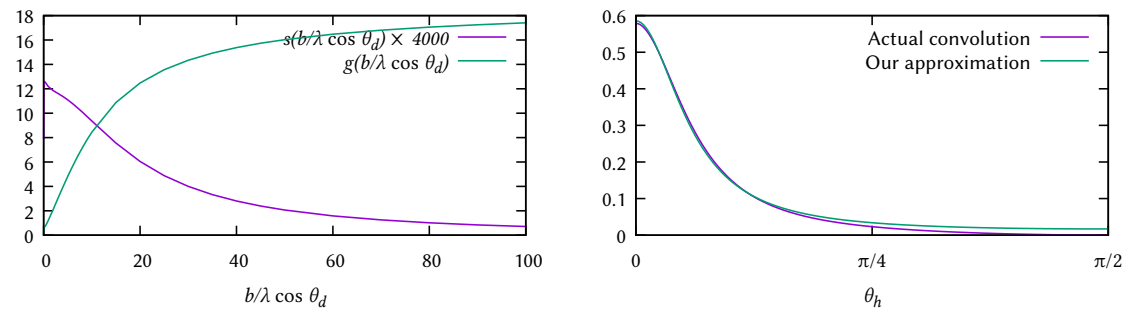

(b) External and internal scaling functions $s$ and $g$ for $\beta=0.03255, p=2.4, c=1.82$. (c) Approximate convolution $(S * D)$ for $\theta_{d}=0$, $\beta=0.0014, p=0.2, \sigma_{s}=0.15, b=1.6, c=1.1$.

Fig. 5. Evaluating the convolution $(S * D)\left(\theta_{h}\right)$ in practical computations.

Diffraction terms in Equation 27 are defined relative to the normal $\boldsymbol{m}$ of the microfacet. We used $\boldsymbol{i} \cdot \boldsymbol{m}$ and $\boldsymbol{o} \cdot \boldsymbol{m}$ in the energy repartition term $A$ in Equation 23. We need to convert these local properties into global properties. For this, we use the fact that $(\boldsymbol{i}+\boldsymbol{o})=2 \cos \theta_{d} \boldsymbol{h}$, where $\boldsymbol{h}$ is the normalized half-vector:

$$
\begin{aligned}
\boldsymbol{i} \cdot \boldsymbol{m} & +\boldsymbol{o} \cdot \boldsymbol{m}=2 \cos \theta_{d}(\boldsymbol{h} \cdot \boldsymbol{m}) \\
\text { also, } f=\|\boldsymbol{f}\| & =2 \cos \theta_{d}\left(1-(\boldsymbol{h} \cdot \boldsymbol{m})^{2}\right)^{\frac{1}{2}} .
\end{aligned}
$$

We approximate $1-A$ using Equation 28:

$$
1-A \approx\left(1-A_{\text {spec }}\left(\theta_{d}\right)\right)(\boldsymbol{h} \cdot \boldsymbol{m})^{2} .
$$

This approximation is exact for extreme values $(\boldsymbol{h} \cdot \boldsymbol{m})=0$ and $(\boldsymbol{h} \cdot \boldsymbol{m})=1$. It is good if $2 \pi\left(2 \cos \theta_{d}\right) \sigma_{\mathrm{rel}} / \lambda<1 / 2$ (see Figure $5 \mathrm{a}$ ).

Two functions in the integrand, $S_{H S}$ and $D$, vary quickly and are almost null over a large part of the interval. We approximate the other functions, which vary more slowly, by their values when $D$ and $S_{H S}$ are maximal, either for $\boldsymbol{m}=\boldsymbol{n}$ or for $f=0$ :

$$
\begin{aligned}
\rho_{C T D} & \approx Q(\boldsymbol{i}, \boldsymbol{o}) G(\boldsymbol{i}, \boldsymbol{o}) \int_{\Omega}(1-A) S_{H S}(\boldsymbol{f}) D(\boldsymbol{m}) \mathrm{d} \omega_{m} \\
& \approx Q(\boldsymbol{i}, \boldsymbol{o}) G(\boldsymbol{i}, \boldsymbol{o})\left(1-A_{\mathrm{spec}}\left(\theta_{d}\right)\right) \times \\
& \int_{\Omega}(\boldsymbol{h} \cdot \boldsymbol{m})^{2} S_{H S}\left(2 \cos \theta_{d}\left(1-(\boldsymbol{h} \cdot \boldsymbol{m})^{2}\right)^{\frac{1}{2}}\right) D(\boldsymbol{m}) \mathrm{d} \omega_{m} .
\end{aligned}
$$

The last integral is a spherical convolution between two functions defined on the sphere: $S(\theta)=S_{H S}\left(2 \cos \theta_{d} \sin \theta\right) \cos ^{2} \theta$ and $D(\theta)$. We use $*$ to denote spherical convolution:

$$
\rho_{C T D} \approx Q(\boldsymbol{i}, \boldsymbol{o}) G(\boldsymbol{i}, \boldsymbol{o})\left(1-A_{\mathrm{spec}}\left(\theta_{d}\right)\right)(S * D)\left(\theta_{h}\right) .
$$

The Cook-Torrance diffraction lobe is, roughly, a spherical convolution between the microfacet normal distribution $D$ and the scattering function of the diffraction lobe times a squared cosine.

To evaluate this convolution, we compute the Spherical Harmonics coefficients of each function, multiply them and compute the inverse transform (see Appendix A). Both functions have rotational symmetry: their Spherical Harmonics decomposition is Zonal Harmonics (coefficients with $m \neq 0$ are null), speeding up computations.

\subsection{Exponential Power Distribution NDF}

For our normal distribution function $D\left(\theta_{h}\right)$, we use the Exponential Power Distribution. It provides a good fit with the sharp decrease near the specular peak that we observe on many measured BRDFs:

$$
\begin{aligned}
D_{E P D}(\theta) & =\frac{\chi_{\left[0, \frac{\pi}{2}\right]}(\theta)}{\cos ^{4} \theta} P_{22}\left(\tan ^{2} \theta\right) \\
P_{22}(x) & =\frac{p}{\pi \beta^{2} \Gamma(1 / p)} \mathrm{e}^{-\left(\frac{x}{\beta^{2}}\right)^{p}} .
\end{aligned}
$$

It has two parameters: $\beta$ controls the width of the peak, while $p$ controls its kurtosis, that is whether it drops sharply at the origin or not. This distribution is similar to the "BRDF model A" obtained by Brady et al. [2014] with genetic programming, but with the shapeinvariant property [Heitz 2014b]. Shape-invariance allows us to compute the Smith shadowing and importance sampling.

We compute the shadowing term $G$ for this distribution using Smith's method [Smith 1967; Walter et al. 2007; Heitz 2014b]:

$$
\begin{aligned}
P_{2}(r) & =\frac{p}{\pi \Gamma(1 / p)} \int_{0}^{\infty} \mathrm{e}^{-\left(r^{2}+q^{2}\right)^{p}} \mathrm{~d} q \\
\Lambda(\beta \tan \theta) & =\int_{1 / \beta \tan \theta}^{\infty}(r \beta \tan \theta-1) P_{2}(r) \mathrm{d} r \\
G_{1}(\theta) & =\frac{1}{1+\Lambda(\beta \tan \theta)} .
\end{aligned}
$$

Because the distribution is shape-invariant, $\Lambda$ can be expressed as a function of $\beta \tan \theta$ and $p$. We precompute $\Lambda$ and store it in a 2D array indexed by $\beta \tan \theta$ and $p$ (a single 2D array for all values of the three parameters $\beta$, $p$, and $\theta$ requires $390 \mathrm{kB}$ of memory).

\subsection{Practical Convolution Computations}

We computed the convolution between $S$ and $D_{E P D}$ for a wide range of parameters, using the K-correlation model for $S_{H S}$. A scaled Kcorrelation model provides a good approximation (see Figure $5 \mathrm{c}$ ):

$$
\begin{aligned}
& (S * D)\left(\theta_{h}\right) \approx s(u, c, \beta, p) S_{H S}\left(f^{\prime}\right) \\
& u=(b / \lambda) \cos \theta_{d} \\
& f^{\prime}=(2 / b) \sin \theta_{h} g(u, c, \beta, p),
\end{aligned}
$$

where $s$ and $g$ are functions that we precompute and store for all values of the parameters. Figure $5 \mathrm{~b}$ displays $s$ and $g$ for a given $(c, \beta, p)$ combination. Below a threshold, $g(u) \approx u$ : convolution with $D$ is just a scaling. Above another threshold, $g(u)$ is almost constant: $(S * D)$ is only a function of $\theta_{h}$, independent of $\theta_{d}$ and $\lambda$. The thresholds depend on $(c, \beta, p)$. Storing the fitted values of $s$ 


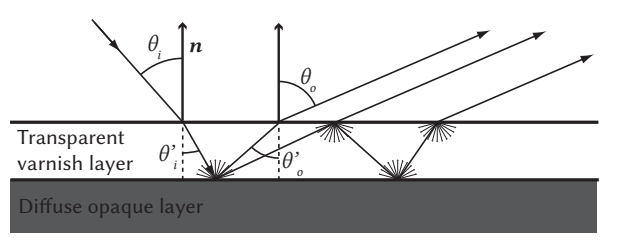

Fig. 6. Our model works with multi-layer materials. In the plastic two-layer model, we assume we have a transparent layer of refractive varnish over a diffuse material. We model light bouncing several times between the two interfaces before leaving the object.

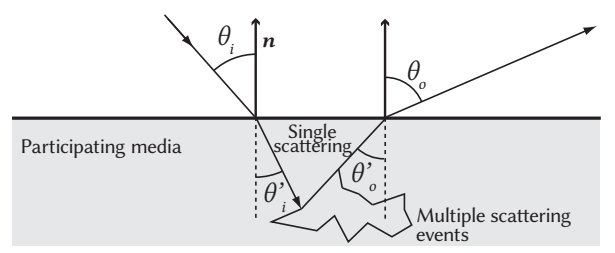

Fig. 7. In the subsurface model, light is reflected on the surface as well as refracted inside the underlying participating media, where it is scattered multiple times before leaving through the refractive interface.

and $g$ for all values of parameters $(c, \beta, p, u)$ requires a $2.5 \mathrm{MB}$ table (provided in companion material).

In practice if $D$ has a sharp peak compared to $S_{H S}, D$ behaves like a Dirac on the convolution $(S * D)$, and $\rho_{C T D}$ is almost the diffraction lobe. If $D$ has a wider peak or $S_{H S}$ has a thinner peak, the convolution has an impact. Close to the specular direction, $\theta_{d}$ is small, so $g$ is constant and $\rho_{C T D}$ only depends on $\theta_{h}$, with no wavelength-based effects. Away from the specular direction, $\theta_{d}$ is large, $\cos \theta_{d}$ is small, $g$ is linear, and we get back to the standard diffraction lobe, with wavelength-dependency.

\subsection{Multi-Layer Models}

Our two-scale BRDF only models interaction at the top material interface. We combine it with multi-layer models, such as plastic (see Figure 6), a transparent layer of varnish over a diffuse layer [Weidlich and Wilkie 2007] or subsurface (see Figure 7), a BRDF approximation of subsurface scattering by participating media [Jensen et al. 2001]

For both models, we model reflection by the top interface using our two-scale model, and refraction into the multi-layer material with specular refraction. The amount of energy passing into the lower layers is $T$ :

$$
T=\left(1-F\left(\theta_{i}\right)\right)\left(1-F\left(\theta_{o}\right)\right),
$$

where $F$ is the Fresnel term for reflection (see Equation 3). For plastic, the reflectance depends on the reflectance $\rho_{d}$ of the diffuse layer:

$$
\rho_{\text {plastic }}=T \frac{\rho_{d}}{\pi}\left(1-\frac{\rho_{d}}{\pi} F_{d r}\left(\frac{1}{\eta}\right)\right)^{-1},
$$

where $F_{d r}$ is the Fresnel diffuse reflectance corresponding to the integral of Fresnel reflectance times the cosine angle over incoming directions.

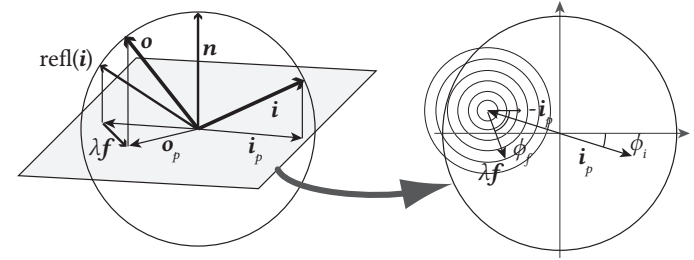

Fig. 8. To importance sample the diffraction lobe: Start with $-\boldsymbol{i}_{p}$, the projection of the reflection of incoming direction $i$. Compute $\lambda f$, the difference between $-\boldsymbol{i}_{p}$ and projected outgoing direction $\boldsymbol{o}_{p}$. Un-project $\boldsymbol{o}_{p}$ to get $\boldsymbol{o}$.

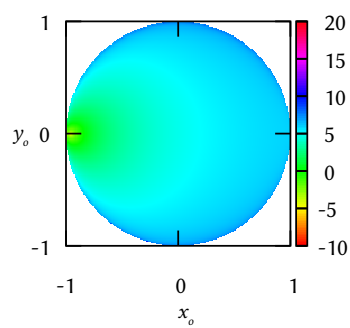

(a) Our model

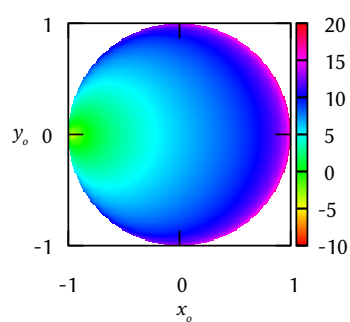

(b) Cook-Torrance alone
Fig. 9. Log of importance weights as a function of $\left(x_{o}, y_{o}\right)$, for $\theta_{i}=1.3$, $\phi_{i}=0$. The diffraction lobe reduces the weights at grazing angles.

For subsurface, it depends on the albedo $\alpha$ of the scattering material:

$$
\begin{aligned}
\text { single } & =\frac{\alpha T}{4 \pi} \frac{1}{\cos \theta_{i}^{\prime}+\cos \theta_{o}^{\prime}} \\
A_{F} & =\frac{1-F_{d r}}{1+F_{d r}} \\
\text { multi } & =\frac{\alpha T}{2 \pi} \mathrm{e}^{-\sqrt{3(1-\alpha)}}\left(1+\mathrm{e}^{-\frac{4}{3} A_{F} \sqrt{3(1-\alpha)}}\right) \\
\rho_{\text {subsurface }} & =\text { single }+ \text { multi, }
\end{aligned}
$$

where $\theta_{i}^{\prime}$ and $\theta_{o}^{\prime}$ are the angles of the refracted rays with the surface normal (see Figure 7).

\subsection{Importance Sampling}

For importance sampling with our model, we select between the Cook-Torrance lobe and the Cook-Torrance diffraction lobe depending on the incoming direction $\boldsymbol{i}$, neglecting convolution effects and renormalization:

$$
A_{\text {imp }}=\mathrm{e}^{-\left(2 \pi \frac{\sigma_{S}}{\lambda} \cos \theta_{i}\right)^{2}}
$$

We take $u_{1}$ a random variable in $[0,1)$ and sample the Cook-Torrance lobe if $u_{1}<A_{\text {imp }}$, the Cook-Torrance-Diffraction lobe otherwise.

To sample the Cook-Torrance lobe, we pick two uniform random variables $\left(u_{2}, u_{3}\right)$ in $[0,1)$ and build a microfacet normal $\boldsymbol{m}$ with $\left(\theta_{m}, \phi_{m}\right)$ :

$$
\begin{aligned}
& \theta_{m}=\arctan \left(\beta\left(\gamma_{u}^{-1}\left(1 / p, u_{3}\right)\right)^{\frac{1}{2 p}}\right) \\
& \phi_{m}=2 \pi u_{2},
\end{aligned}
$$



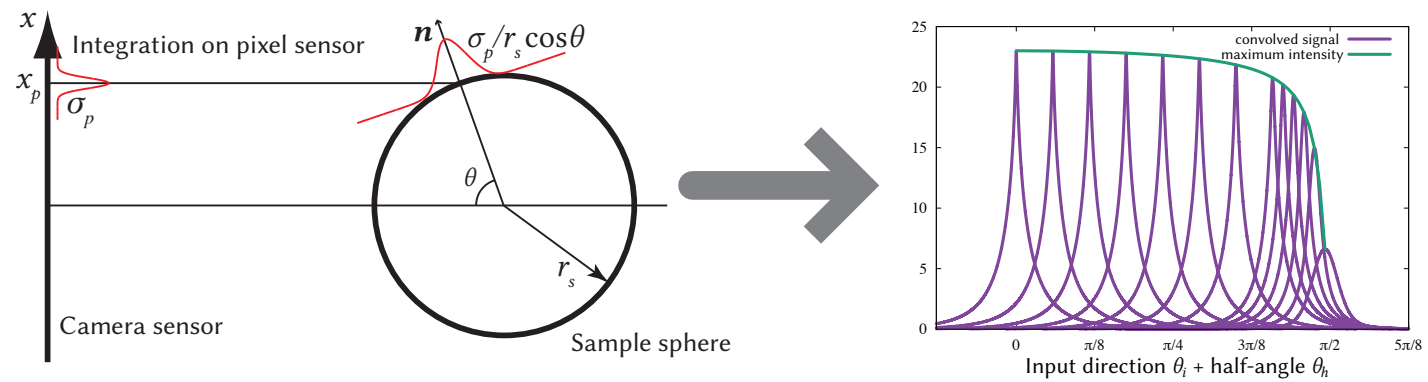

Fig. 10. (left) BRDFs in the MERL database were acquired using photographs of sample spheres. Each camera pixel performs an integral over a small spatial domain of width $\sigma_{p}$. Taking photographs of a sphere of radius $r_{s}$, this translates into a convolution between the BRDF and a low-pass filter of width $\sigma_{p} /\left(r_{s} \cos \theta\right)$. (right) We visualize the effects of this convolution on the distribution $D\left(\theta_{h}\right)$ over varying $\theta_{i}$ : a reduction in frequency and the maximum intensity decreases at grazing angles.

where $\gamma_{u}^{-1}$ is the inverse of the normalized upper incomplete gamma function. The outgoing direction $\boldsymbol{o}$ is the specular reflection of $\boldsymbol{i}$ with respect to $\boldsymbol{m}$. The PDF is: $A_{\text {imp }} D\left(\theta_{h}\right) \cos \theta_{h} /\left(4 \cos \theta_{d}\right)$.

We use a similar method to sample the Diffraction lobe: first build the vector $f$ connecting $\operatorname{refl}(\boldsymbol{i})$ and $\boldsymbol{o}$, then extract $\boldsymbol{o}$. All computations take place in the unit disc, projection of the unit sphere of directions (see Figure 8). $\boldsymbol{i}_{p}$ and $\boldsymbol{o}_{p}$ are the projections of $\boldsymbol{i}$ and $\boldsymbol{o}$ respectively. Using uniform random variables $\left(u_{2}, u_{3}\right)$ in $[0,1)$, we build $f$ in polar coordinates $\left(f, \phi_{f}\right)$ :

$$
\begin{gathered}
f=\frac{1}{b} \sqrt{\left(1-M u_{2}\right)^{-\frac{2}{c-1}}-1} \\
\text { where } M=1-\left(1+\frac{b^{2}}{\lambda^{2}}\left(1+\sin \theta_{i}\right)^{2}\right)^{-\frac{c-1}{2}} .
\end{gathered}
$$

This gives $f \in\left[0,\left(1+\sin \theta_{i}\right) / \lambda\right]$. We pick $\phi_{f}$ so that $\boldsymbol{o}_{p}$ remains inside the unit disc:

$$
\begin{aligned}
\phi_{\max } & =\arccos \left(\max \left(-1, \frac{f^{2}+\sin ^{2} \theta_{i}-1}{2 f \sin \theta_{i}}\right)\right) \\
\phi_{f} & =\phi_{i}+\pi+\left(2 u_{3}-1\right) \phi_{\max } \\
\boldsymbol{o}_{p} & =-\boldsymbol{i}_{p}+\lambda \boldsymbol{f} .
\end{aligned}
$$

This sampling does not provide an equal coverage of the unit disc; each sample must be weighted by its inverse probability, $\phi_{\max } / \pi$. The PDF is: $\left(1-A_{\text {imp }}\right) S_{H S}(f) \cos \theta_{o} \pi / \phi_{\max }$. All pictures in this paper are generated using importance sampling. Importance weights, defined as the inverse of the PDF, can become quite large at grazing angles (see Figure 9a). This effect is less pronounced with our model than when using a Cook-Torrance lobe alone (see Figure 9b).

\section{VALIDATION WITH MEASURED MATERIALS}

To test the validity of our reflectance model combining reflection and diffraction, we used it to fit the 100 materials in the MERL database [Matusik et al. 2003]. We searched for optimal parameters using Levenberg-Marquadt optimisation, as implemented by Lourakis [2004]. We conduct our fitting over the entire parameter domain $\left(\theta_{i}, \theta_{o}, \phi_{o}-\phi_{i}\right)$, using an $L^{2}$ norm:

$E^{2}=\int(\operatorname{measured}(\boldsymbol{i}, \boldsymbol{o})-p(\boldsymbol{i}, \boldsymbol{o}) \operatorname{predicted}(\boldsymbol{i}, \boldsymbol{o}))^{2} w_{C} \mathrm{~d} \omega_{\boldsymbol{o}} \cos \theta_{i} \mathrm{~d} \theta_{i}$.
We introduced two changes: First, we use the compressive weight $w_{C}$ from Bagher et al. [2016]. It strongly improves the stability of the fitting process, preventing it from trying too hard to fit higher values, more likely to contain larger errors:

$$
\begin{aligned}
& w_{C}=\left(\frac{f(\text { measured }(\boldsymbol{i}, \boldsymbol{o}) / \text { median, } \log 2)}{\text { measured }(\boldsymbol{i}, \boldsymbol{o}) / \text { median }}\right)^{p} \\
& f(x, \alpha)=1 / \alpha\left(1-\mathrm{e}^{-\alpha x}\right),
\end{aligned}
$$

where median is the median value of the measured data, weighted by $\cos \theta_{i}$; for $p$, we used $p=1.4$.

Second, we compensate for errors introduced at grazing angles by the MERL acquisition apparatus with $p(\boldsymbol{i}, \boldsymbol{o})$. Matusik et al. [2003] used spheres to speed up the acquisition process: a single photograph covers all outgoing directions. For each pixel, a camera sensor integrates over a small spatial domain, operating a spatial convolution between the pixel footprint and the incoming signal. For a photograph of a sphere, this translates into an angular convolution between the BRDF and the pixel footprint with its width divided by $\cos \theta$ (see Figure 10 and supplemental material).

This convolution has two effects, visible on Figure 10, right: it lowers the frequency content of the signal, and reduces its maximum intensity. Assuming pixel response is a Gaussian, we computed the latter effect and approximate it with:

$$
\begin{aligned}
& p(\boldsymbol{i}, \boldsymbol{o})=p_{1}\left(\theta_{i}\right) p_{1}\left(\theta_{o}\right) \\
& p_{1}(\theta)=\left(1-\frac{4 \theta^{2}}{\pi^{2}}\right)^{k(\beta, p)} .
\end{aligned}
$$

The exponent $k$ depends on the BRDF frequency content. For a glossy or diffuse material, with low frequency effects, $k \approx 0$ : the acquisition did not distort the signal. For a specular material, $k \approx 1$ : there is a significant reduction in signal intensity at grazing angles. This effect has been observed before [Ngan et al. 2005]. We show it comes from the acquisition apparatus and provide a model for it, which we use in our fitting process.

\subsection{Results and Comparison}

We compare how our model approximates measured materials to four reference models: Shifted-Gamma Distribution (SGD) [Bagher et al. 2012], Smooth reflectance model [Löw et al. 2012], He model [He 
Table 1. The models used in the comparison. EPD $=$ Exponential Power Distribution (see Section 4.3), $S G D=$ Shifted-Gamma Distribution, $A B C=K$-correlation model (see Section 3.2.3).

\begin{tabular}{lccccccl}
\hline Name & Reference & Based on & Lobe shape & $\begin{array}{c}\text { Physically } \\
\text { consistent }\end{array}$ & $\begin{array}{c}\text { Nb. } \\
\text { param. }\end{array}$ & $\begin{array}{c}\text { Complex } \\
\text { IOR }\end{array}$ & $\begin{array}{l}\text { Origin of the } \\
\text { parameter values }\end{array}$ \\
\hline Ours & & CT + Diff & EPD \& ABC & yes & 11 & yes & our fitting \\
EPD & & Cook-Torr. & EPD & yes & 11 & yes & our fitting \\
SGD & [Bagher et al. 2012] & Cook-Torr. & SGD & no & 18 & no & supplemental of [Bagher et al. 2012] \\
He & [He et al. 1991] & Diffraction & Gaussian & yes & 11 & yes & our fitting \\
Smooth & [Löw et al. 2012] & Diffraction & ABC & no & 9 & no & supplemental of [Löw et al. 2012] \\
\hline
\end{tabular}

Table 2. Metrics used for comparison

sMAPE: Symmetric Mean Absolute Percentage Error

$$
E_{\text {SMAPE }}=\frac{\left|V_{\text {measured }}-V_{\text {predicted }}\right|}{V_{\text {measured }}+V_{\text {predicted }}} \text {. }
$$

RMSE: Root-Mean Square Error

$$
E_{\mathrm{RMSE}}\left(\theta_{i}\right)=\left(\int(\rho(\boldsymbol{i}, \boldsymbol{o})-f(\boldsymbol{i}, \boldsymbol{o}))^{2} \cos ^{2} \theta_{i} \mathrm{~d} \omega_{\boldsymbol{o}}\right)^{\frac{1}{2}} \text {. }
$$

et al. 1991] and a Cook-Torrance model with the Exponential Power Distribution (EPD). These four models cover a wide range of properties and representations (see Table 1). We use several metrics for model comparison: visual image difference, average image difference using sMAPE, BRDF energy difference using RMSE, and lobe shape, both inside and outside the incident plane (see Table 2). The supplemental material provides these metrics for all materials in the MERL database.

SGD and Smooth models disagree with the underlying physical model: SGD is based on Cook-Torrance, but has channel-dependent NDF parameters, implying that the micro-geometry is different for each color channel. The Smooth model is based on diffraction, but without wavelength dependency and renormalization.

5.1.1 Comparison between Models: All models provide a good approximation of measured data, with a few spectacular exceptions. Models based on diffraction alone (He, Smooth) provide a good description of diffuse and glossy materials. Models based on CookTorrance (our model, EPD, SGD) tend to look better on shiny and specular materials.

Looking at both qualitative (image comparison) and quantitative metrics (sMAPE, RMSE), our model provides a very good representation for glossy and specular materials. It is good, but not as much as other models for diffuse and almost diffuse materials, such as fabrics. These diffuse materials break several assumptions of the microfacet model; taking into account multiple scattering effects could increase quality.

Specifically, our model has a smaller RMSE than other models for 62 materials, corresponding to all glossy to specular materials, from silver-paint to chrome (see Figure 11). On these, there is almost no difference left between the measured data and what our model predicts (see Figure 12).

Interestingly, our model also provides a very good approximation for some materials where its average RMSE is larger than for the
Smooth or SGD models. It can happen that our model provides a better fit with the measured data and lobe shape everywhere except at grazing angles (see Figure 13).

5.1.2 Lobe Shapes: Our model excels at predicting lobe shapes for reflectance lobes. Both inside the incident plane (see Figures 12 and 13) and outside the incident plane. We represent these as isolines on the unit sphere (see Figure 14). As pointed by Löw et al. [2012], outside the specular peak, the diffraction model provides a better prediction of lobe shape. We improve on their fitting, especially at grazing angles, by taking into account the interconnection between micro-geometry and diffraction geometry.

5.1.3 Importance of the Diffraction Lobe: The relative intensities of diffraction effects and Cook-Torrance effects depend on the material roughness: For smooth materials (e.g. chrome) diffraction effects have smaller intensity than Cook-Torrance effects. The reverse is true for glossy materials (blue-metallic-paint, gold-metallic-paint). Either way, diffraction effects play an important role in both the visual aspect and the fitting process. The EPD model has the same number of parameters as our model, with an independent diffuse lobe. Our model provides a better approximation on most materials.

Lobe width can change with color channel (see Figure 15), in contradiction with the microfacet model. Our model explains and predicts this variation, caused by a strong diffraction lobe. Butler et al. [2015b] observe a similar effect in their measures.

Several materials previously thought to require multiple lobes for fitting are well approximated by our model: red-metallic-paint, alum-bronze, two-layer-gold; one of the lobe corresponds to the microfacet lobe, the other to diffraction effects. Both lobes have the same index of refraction.

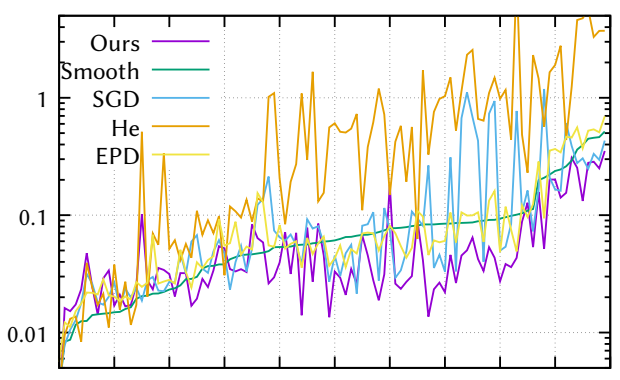

Fig. 11. RMSE on the BRDF for all materials in the MERL database. 
For some materials (e.g. gold-metallic-paint3, specular-orange-phenolic) it is hard to separate the respective contributions of the Cook-Torrance, diffraction and diffuse components. For these, the fitting process places all contributions in one of the lobes. This gives a good visual and numerical fit, but not a good representation of the physical phenomena.

5.1.4 Connection with Material Names: There is a strong correspondence between the name of a material and the best model to approximate it: materials with plastic, phenolic or acrylic in their names are well approximated by the plastic model, materials named after stones are well approximated by the subsurface model (e.g. aventurine, white-marble). For most metallic paints, we found a better fit using the plastic model, but with a dark diffuse layer. This could be related to multiple scattering effects inside the microgeometry, or to a different material model, e.g. metallic flakes in a semi-transparent primer [Ergun et al. 2016].

\subsection{Editing Parameters}

Our model has two different sets of parameters. Roughness $\beta$ and kurtosis $p$ control the Cook-Torrance lobe. $\sigma_{s}, b$ and $c$ control the diffraction lobe: $\sigma_{s}$ is the standard deviation of nano-geometry; $b$ is the inverse of a length. To increase diffraction effects, we increase $\sigma_{s}$ and decrease $b$. Figure 16 shows the effects of multiplying $\sigma_{s}$ and dividing $b$ by identical amounts, increasing diffraction effects while keeping Cook-Torrance effects unchanged. Figure 17 shows the reverse: changing roughness $\beta$ while keeping other parameters unchanged. Comparing these two figures, increasing roughness has an effect on the entire sphere, making it appear more diffuse, while increasing diffraction has an impact on the center of the sphere, making it appear more glazy.

\section{CONCLUSION AND FUTURE WORK}

We have presented a new material reflectance model, based on a two-scale representation of surface micro-geometry. Our model takes into account diffraction effects caused by smaller-scale details. These effects are combined together by the larger scale geometry, and added to the standard Cook-Torrance reflection over the larger scale geometry. This model provides a good approximation with measured materials, including at grazing angles or away from the specular peak. Our model also explains the wavelength dependency observed in measured materials.

In future work, we want to extend this two scale approach combining diffraction and Cook-Torrance to multi-layer materials [Jakob et al. 2014] and multiple scattering [Heitz et al. 2016]. We also plan to use this model for faster acquisition of measured reflectance.

\section{A PRACTICAL CONVOLUTION}

To compute the spherical convolution $(S * D)$, we:

- compute the Spherical Harmonics coefficients of each function. Since both functions have rotational symmetry around the $z$ axis, only SH coefficients with $m=0$ are non-null (i.e., Zonal Harmonics):

$$
\begin{aligned}
& Y_{\ell}^{0}(\theta, \varphi)=\sqrt{\frac{2 \ell+1}{4 \pi}} P_{\ell}(\cos \theta) \\
& S_{\ell}=\int_{\Omega} S(\theta) Y_{\ell}^{0}(\theta) \mathrm{d} \omega=2 \pi \int_{\theta} S(\theta) Y_{\ell}^{0}(\theta) \sin \theta \mathrm{d} \theta \\
& D_{\ell}=\int_{\Omega} D(\theta) Y_{\ell}^{0}(\theta) \mathrm{d} \omega=2 \pi \int_{\theta} D(\theta) Y_{\ell}^{0}(\theta) \sin \theta \mathrm{d} \theta
\end{aligned}
$$

where $P_{\ell}$ is the Legendre polynomial of degree $\ell$.

- multiply them to get the Spherical Harmonics coefficients of the convolution, and extract the function:

$$
(S * D)(\theta, \varphi)=\sum_{\ell} S_{\ell} D_{\ell} \sqrt{\frac{4 \pi}{2 \ell+1}} Y_{\ell}^{0}(\theta, \varphi)
$$

We ran precomputations of $(S * D)$ with 100 Zonal Harmonics coefficients, for all values of the parameters, and approximate the result with a scaled K-correlation model (see Equations 39 to 41).

\section{REFERENCES}

M. Ashikhmin and S. Premože. 2007. Distribution-based BRDFs. University of Utah. (2007). http://www.cs.utah.edu/ premoze/dbrdf/.

M. M. Bagher, J. Snyder, and D. Nowrouzezahrai. 2016. A Non-Parametric Factor Microfacet Model for Isotropic BRDFs. ACM Trans. Graph. 36, 5, Article 159 (July 2016), 16 pages. DOI : https://doi.org/10.1145/2907941

M. M. Bagher, C. Soler, and N. Holzschuch. 2012. Accurate fitting of measured reflectances using a Shifted Gamma micro-facet distribution. Computer Graphics Forum 31, 4 (June 2012). DOI : https://doi.org/10.1111/j.1467-8659.2012.03147.x

P. Beckmann and A. Spizzichino. 1987. The scattering of electromagnetic waves from rough surfaces. Artech House.

A. Brady, J. Lawrence, P. Peers, and W. Weimer. 2014. genBRDF: Discovering New Analytic BRDFs with Genetic Programming. ACM Trans. Graph. 33, 4, Article 114 (July 2014), 11 pages. DOI : https://doi.org/10.1145/2601097.2601193

B. Burley. 2012. Physically-Based Shading at Disney. In Siggraph course: Practical Physically Based Shading in Film and Game Production, Stephen Hill and Stephen McAuley (Eds.). ACM. https://disney-animation.s3.amazonaws.com/library/s2012_ pbs_disney_brdf_notes_v2.pdf

S. D. Butler, S. E. Nauyoks, and M. A. Marciniak. 2015a. Experimental analysis of bidirectional reflectance distribution function cross section conversion term in direction cosine space. Opt. Lett. 40, 11 (Jun 2015), 2445-2448. DOI : https://doi.org/ 10.1364/OL.40.002445

S. D. Butler, S. E. Nauyoks, and M. A. Marciniak. 2015b. Experimental measurement and analysis of wavelength-dependent properties of the BRDF. Proc. SPIE 9611, Imaging Spectrometry XX (2015). DOI : https://doi.org/10.1117/12.2188093

E. L. Church and P. Z. Takacs. 1986. Statistical And Signal Processing Concepts In Surface Metrology. (1986). DOI: https://doi.org/10.1117/12.964495

R. L. Cook and K. E. Torrance. 1982. A Reflectance Model for Computer Graphics. ACM Trans. Graph. 1, 1 (1982), 7-24. DOI : https://doi.org/10.1145/357290.357293

S. Ergun, S. Önel, and A. Ozturk. 2016. A General Micro-flake Model for Predicting the Appearance of Car Paint. In Eurographics Symposium on Rendering - EI \& I. DOI : https://doi.org/10.2312/sre.20161211

J. E. Harvey. 1975. Light-Scattering Characteristics of Optical Surface. Ph.D. Dissertation. University of Arizona. http://www.dtic.mil/dtic/tr/fulltext/u2/a095132.pdf Adviser R. V. Shack.

J. E. Harvey, S. Schröder, N. Choi, and A. Duparré. 2012. Total integrated scatter from surfaces with arbitrary roughness, correlation widths, and incident angles. Optical Engineering 51, 1 (2012). DOI : https://doi.org/10.1117/1.OE.51.1.013402

X. D. He, K. E. Torrance, F. X. Sillion, and D. P. Greenberg. 1991. A Comprehensive Physical Model for Light Reflection. Computer Graphics (ACM SIGGRAPH '91 Proceedings) 25, 4 (July 1991), 175-186.

E. Heitz. 2014a. Multi-scale appearance for realistic and efficient rendering of complex surfaces. Ph.D. Dissertation. Université de Grenoble. https://tel.archives-ouvertes. fr/tel- 01073518

E. Heitz. 2014b. Understanding the Masking-Shadowing Function in Microfacet-Based BRDFs. Fournal of Computer Graphics Techniques 3, 2 (June 2014), 32-91. http: //jcgt.org/published/0003/02/03/

E. Heitz, J. Hanika, E. d'Eon, and C. Dachsbacher. 2016. Multiple-Scattering Microfacet BSDFs with the Smith Model. ACM Trans. Graph. (Proc. SIGGRAPH 2016) 35, 4, Article 58 (July 2016). DOI : https://doi.org/10.1145/2897824.2925943 


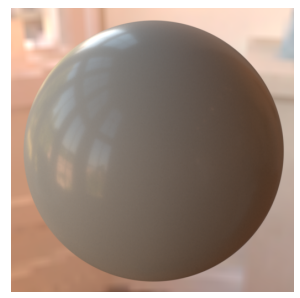

Smooth

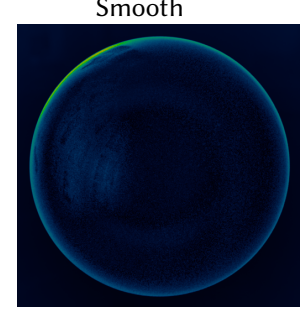

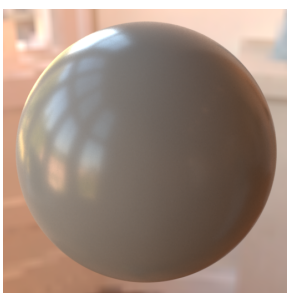

SGD

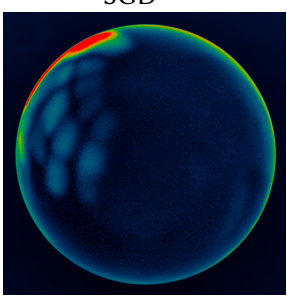

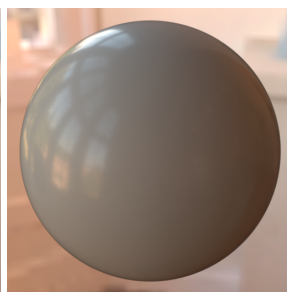

Our model

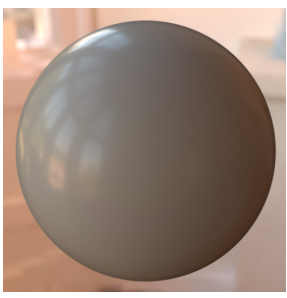

Reference

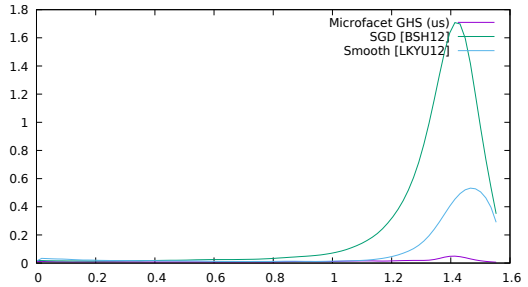

RMSE as a function of $\theta_{i}$
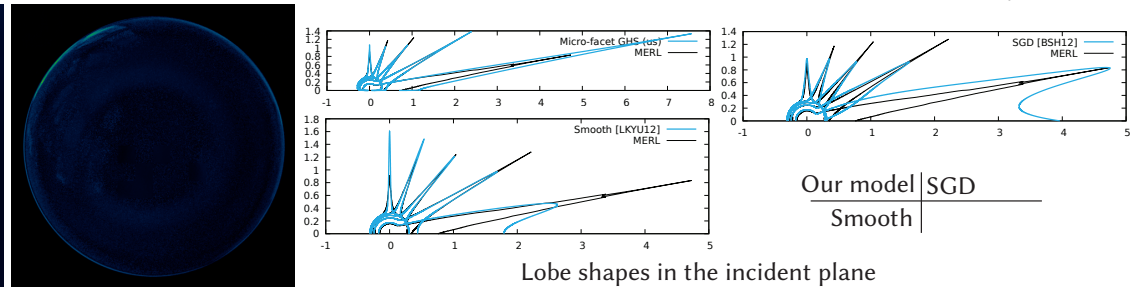

Fig. 12. A material where our model performs well: gray-plastic. Material behaviour predicted by our model is extremely close to measured data. Difference images use the Lab color space.

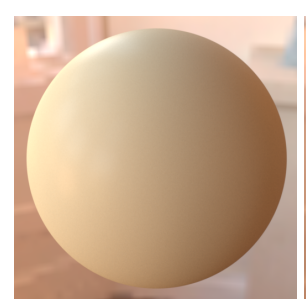

Smooth

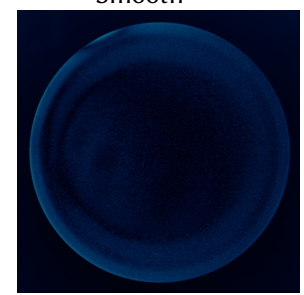

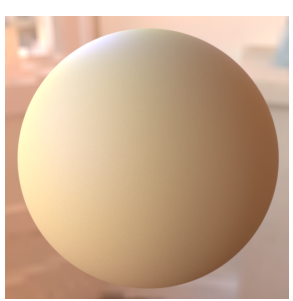

SGD

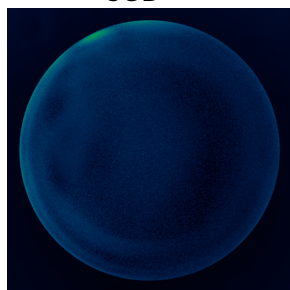

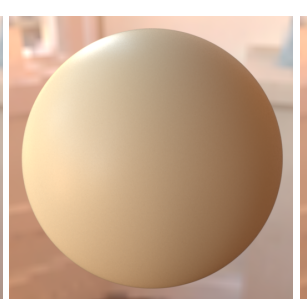

Our model

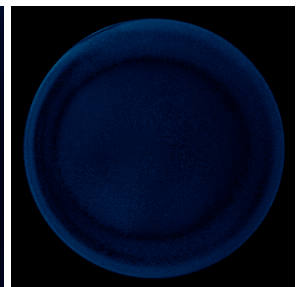

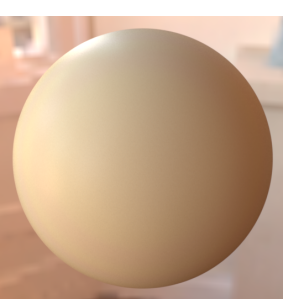

Reference

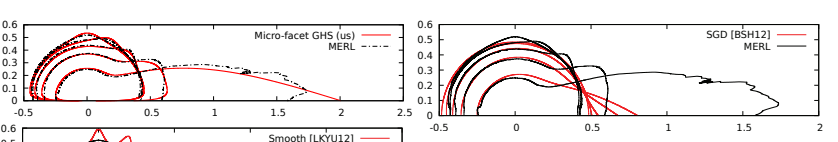

Our model SGD

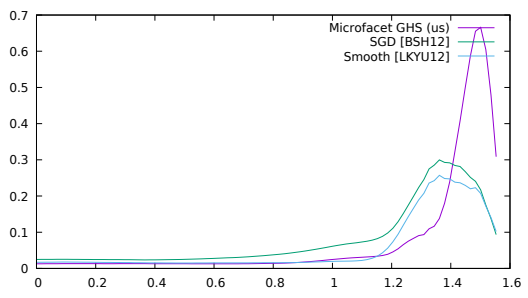

RMSE as a function of $\theta_{i}$

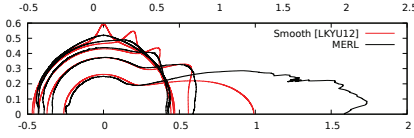

Lobe shapes in the incident plane

Fig. 13. A material where RMSE for our model is larger than for others: white-diffuse-bball. Images and lobe shapes generated by our model are actually closer to the reference, except at grazing angles. Difference images use the Lab color space.

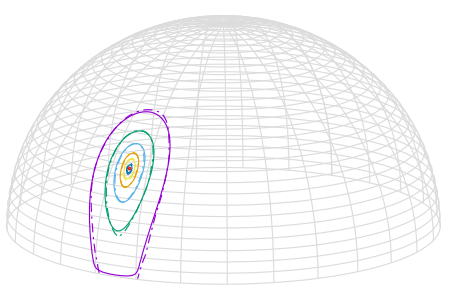

(a) Our model

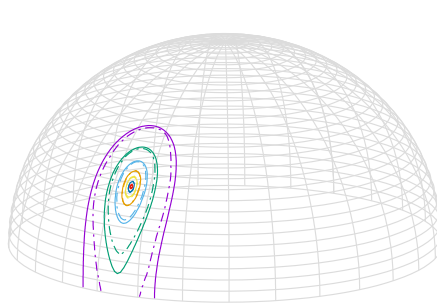

(b) Smooth BRDF model

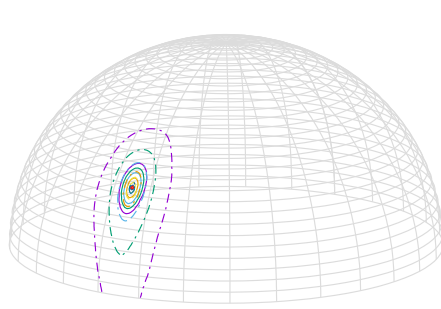

(c) SGD model

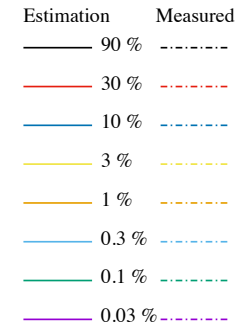

Fig. 14. Lobe shapes for blue-metallic-paint2 (blue channel) outside the incident plane, visualized as isolines: our model (a) provides a striking representation of BRDF behavior at all angles. The Smooth BRDF (b) model provides a good approximation but the quality decreases at grazing angles. The SGD model (c) does not have the correct behavior for low values of the BRDF. 


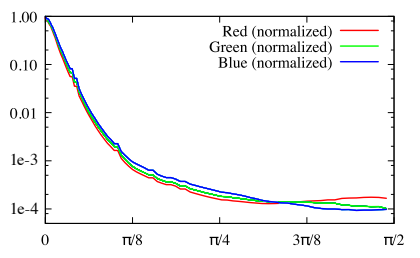

(a) Together

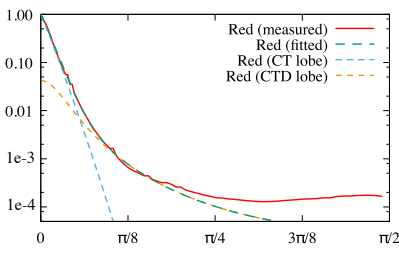

(b) Red

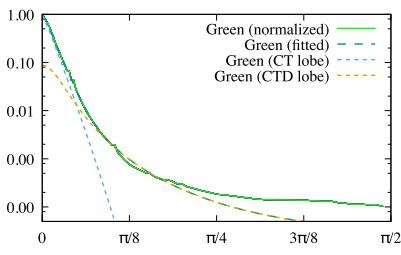

(c) Green

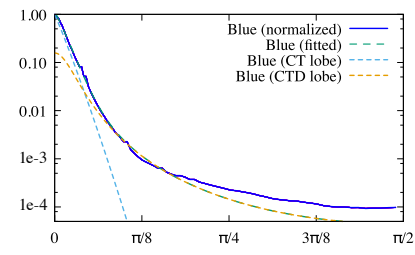

(d) Blue

Fig. 15. (a) Measured reflectance data for nickel as a function of $\theta_{h}$, renormalized by value at origin. The width of the BRDF lobe changes with the wavelength, in contradiction with the microfacet theory. (b-d): The presence of a diffraction lobe, combined with the reflection, explains this variation of lobe width. The diffraction lobe intensity increases as the wavelength decreases (from red to blue).

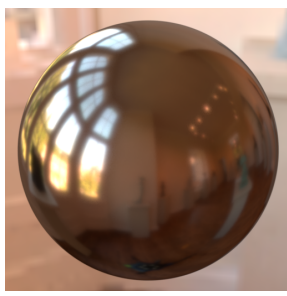

$\sigma_{s} / 4, b \times 4$

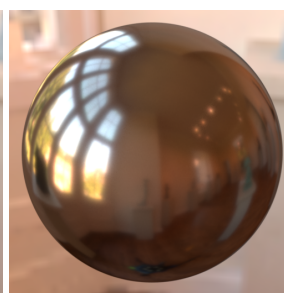

$\sigma_{s} / 2, b \times 2$

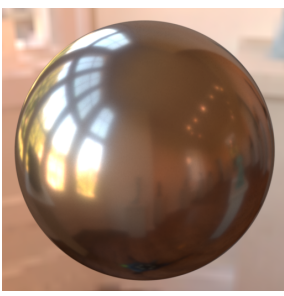

$\sigma_{s} \times 1, b \times 1$

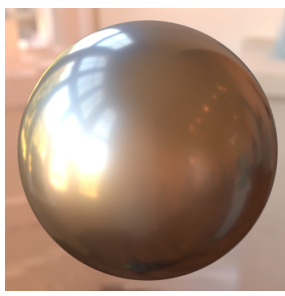

$\sigma_{s} \times 2, b / 2$

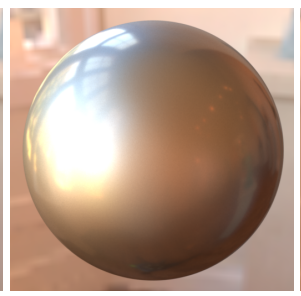

$\sigma_{s} \times 3, b / 3$

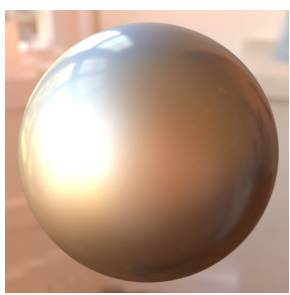

$\sigma_{s} \times 4, b / 4$

Fig. 16. Changing diffraction parameters $\sigma_{s}$ and $b$. Parameters are relative to values fitted for measured nickel. For all spheres, $\beta=0.0598$ ( $\beta / 2$ in Figure 17 ).

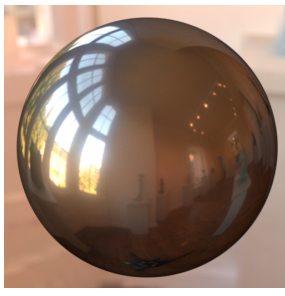

$\beta / 8$

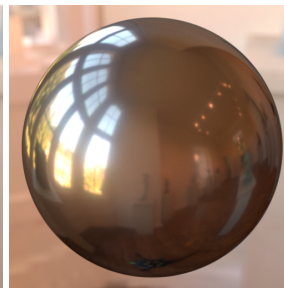

$\beta / 4$

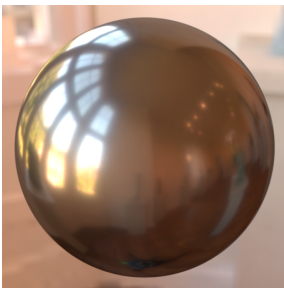

$\beta / 2$

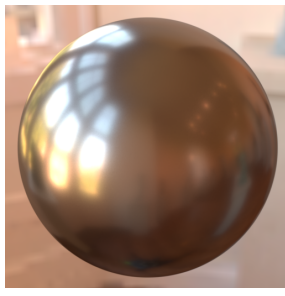

$\beta \times 1$

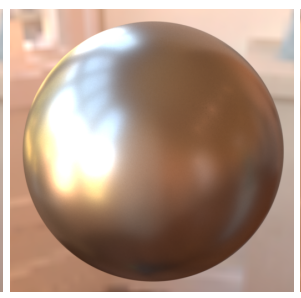

$\beta \times 2$

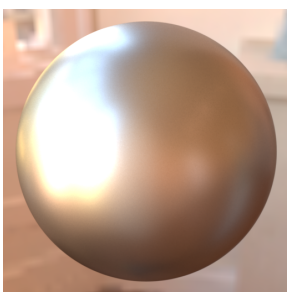

$\beta \times 3$

Fig. 17. Changing roughness parameter $\beta$. Parameters are relative to values fitted for measured nickel. For all spheres, $b=4.8418 \mu \mathrm{m}^{-1}, \sigma_{s}=0.0273 \mu \mathrm{m}$ $\left(\sigma_{s} \times 1, b \times 1\right.$ in Figure 16).

B. J. Hoenders, E. Jakeman, H. P. Baltes, and B . Steinle. 1979. K Correlations and Facet Models in Diffuse Scattering. Optica Acta: International Journal of Optics 26, 10 (1979), 1307-1319. DOI : https://doi.org/10.1080/713819894

N. Holzschuch and R. Pacanowski. 2015a. A physically accurate reflectance model combining reflection and diffraction. Research Report RR-8807. INRIA. https://hal. inria.fr/hal-01224702

N. Holzschuch and R. Pacanowski. 2015b. Identifying diffraction effects in measured reflectances. In Eurographics Workshop on Material Appearance Modeling. https //hal.inria.fr/hal-01170614

N. Holzschuch and R. Pacanowski. 2016. A Physically-Based Reflectance Model Combining Reflection and Diffraction. Research Report RR-8964. INRIA. https: //hal.inria.fr/hal-01386157

W. Jakob, E. D’Eon, O. Jakob, and S. Marschner. 2014. A Comprehensive Framework for Rendering Layered Materials. ACM Trans. Graph. (Proc. SIGGRAPH 2014) 33, 4 (2014). DOI: https://doi.org/10.1145/2601097.2601139

H. W. Jensen, S. Marschner, M. Levoy, and P. Hanrahan. 2001. A Practical Model for Subsurface Light Transport. In SIGGRAPH 2001. 511-518. DOI : https://doi.org/10. $1145 / 383259.383319$

A. Krywonos. 2006. Predicting Surface Scatter using a Linear Systems Formulation of Non-Paraxial Scalar Diffraction. Ph.D. Dissertation. University of Central Florida. http://etd.fcla.edu/CF/CFE0001446/Krywonos_Andrey_200612_PhD.pdf Adviser: J. E. Harvey.

E. P. Lafortune, S.-C. Foo, K. E. Torrance, and D. P. Greenberg. 1997. Non-linear approximation of reflectance functions. In SIGGRAPH '97. 117-126. DOI : https: //doi.org/10.1145/258734.258801

M. I. A. Lourakis. 2004. levmar: Levenberg-Marquardt nonlinear least squares algorithms in C/C++. http://www.ics.forth.gr/ lourakis/levmar/. (July 2004).
J. Löw, J. Kronander, A. Ynnerman, and J. Unger. 2012. BRDF models for accurate and efficient rendering of glossy surfaces. ACM Trans. Graph. 31, 1, Article 9 (Feb. 2012), 14 pages. DOI : https://doi.org/10.1145/2077341.2077350

W. Matusik, H. Pfister, M. Brand, and L. McMillan. 2003. A Data-Driven Reflectance Model. ACM Trans. Graph. 22, 3 (2003). DOI : https://doi.org/10.1145/882262.882343

A. Ngan, F. Durand, and W. Matusik. 2005. Experimental Analysis of BRDF Models. In Eurographics Symposium on Rendering. DOI : https://doi.org/10.2312/EGWR/EGSR05/ 117- 126

B. Smith. 1967. Geometrical shadowing of a random rough surface. IEEE Transactions on Antennas and Propagation 15, 5 (Sept. 1967). DOI : https://doi.org/10.1109/TAP. 1967.1138991

J. Stam. 1999. Diffraction Shaders. In SIGGRAPH '99. ACM, 101-110. DOI : https://doi. org $/ 10.1145 / 311535.311546$

K. E. Torrance and E. M. Sparrow. 1967. Theory for Off-Specular Reflection From Roughened Surfaces. 7. Opt. Soc. Am. 57, 9 (Sept. 1967), 1105-1112. DOI : https: //doi.org/10.1364/JOSA.57.001105

T. S. Trowbridge and K. P. Reitz. 1975. Average irregularity representation of a rough surface for ray reflection. F. Opt. Soc. Am. 65, 5 (1975), 531-536. DOI : https://doi.org/ 10.1364/JOSA.65.000531

C. L. Vernold and J. E. Harvey. 1998. A modified Beckmann-Kirchhoff scattering theory for nonparaxial angles. Proc. SPIE 3426, Scattering and Surface Roughness II (1998), 51-56. DOI : https://doi.org/10.1117/12.328477

B. Walter, S. R. Marschner, H. Li, and K. E. Torrance. 2007. Microfacet models for refraction through rough surfaces. In Eurographics Symposium on Rendering. 195206. DOI : https://doi.org/10.2312/EGWR/EGSR07/195-206

A. Weidlich and A. Wilkie. 2007. Arbitrarily layered micro-facet surfaces. In GRAPHITE '07. 171-178. DOI : https://doi.org/10.1145/1321261.1321292 